\title{
Current Treatment Regimen for Breast Cancer
}

\section{Prathyoosh C* and Karukakulam Sandra Dominic}

Department of Pharmacy Practice, NITTE Deemed to be University, Mangalore, Karnataka, India

*Corresponding Author: Prathyoosh C, Department of Pharmacy Practice, NITTE Deemed to be University, Mangalore, Karnataka, India.
Received: March 30, 2020

Published: June 30, 2020

(C) All rights are reserved by Prathyoosh C and Karukakulam Sandra Dominic.

\begin{abstract}
Breast cancer is one of the most commonly diagnosed cancer alongside the lung cancer and colorectal cancer, and the fourth leading cause of cancer-related deaths in worldwide. One of the major challenges for breast cancer treatment is its versatile nature, which selects the therapeutic options.

BC mainly categorized into 3 subtypes based on estrogen or progesterone receptor expression and HER2 expression. The selection of systematic therapy is based on the presence or expression of the receptors and DNA mutation. Triple negative BC is hard to treat because of the lack of expression [1].

Keywords: Breast Cancer; New Chemotherapy Regimen; HR Positive Breast Cancer; HER2 Negative; HER2 Positive; Triple Negative
\end{abstract}

\section{Abbreviations}

BC: Breast Cancer; HR: Hormone Receptor; HER2: Hormone Response Element; TNBC: Triple Negative Breast Cancer

\section{Introduction}

Breast cancer is one of the most commonly diagnosed cancer alongside the lung cancer and colorectal cancer, and the fourth leading cause of cancer-related deaths in worldwide. One of the major challenges for breast cancer treatment is its versatile nature, which selects the therapeutic options [1]. Breast cancer is classified into four sub types according to its molecular characters which are, (1) luminal A (HR+/HER2), (2) HER2+, (3) luminal B $(\mathrm{HR}+/ \mathrm{HER} 2+)$ and (4) triple negative (TNBC; HR-/HER2-; also overlap with the basal-like subtype). Each of these subtypes are different in terms of its risk factors for incidence, therapeutic response, disease progression and preferential organ sites of metastases [2].

Breast cancer can be diagnosed with different procedure which includes, mammogram and its sub classification, biopsy, physical examination, ultrasound and breast MRI. And for staging the disease along with these procedures blood test, bone scan, CT scan and PCT scan can be carried out [3].

\section{Materials and Methods}

Conducted a search in PubMed and other sources for Englishlanguage articles related to the treatment of breast cancer form the year 2016. The indices of major medical and oncology journals were comprehensively reviewed and established a result [3].

\section{Results and Discussion}

Current treatment regimen for breast cancer

HR-positive breast cancer treatment

Targeted therapy is the method of using drugs and/or other substances to act on the cancer cells and produces the desired ef- fect with less harm to normal cells. There is a new plan of adding targeted therapies and hormone therapy for advanced metastatic HR-positive breast cancer. These treatments will help to prolong the time until chemotherapy is required and ideally, with extend survival rate.

Drugs used in this include, Palbociclib (Ibrance), ribociclib (Kisqali), and everolimus (Afinitor) which all have been approved by the FDA recently to use with hormonal.

Abemaciclib (Verzenio) can be given with or after hormonal therapy to women with advanced metastatic, with ER positive, HRE2 negative. These combinations can prevent a relapse after treatment of early-stage ER-positive disease which was tested and conformed.

Alpelisib (Piqray) can be used for in combination with hormonal therapy to treat HR-positive and HER2-negative breast cancers that have a mutation in the PIK3CA gene.

\section{HER2-positive breast cancer treatment}

There are a number of combination which is approved by FDA, which includes: Trastuzumab (Herceptin) and pertuzumab (Perjeta) in alone or combination can be used with chemotherapy for both early stage and advanced breast cancer. Trastuzumab got approved for use to prevent a relapse in patients with early HER2positive breast cancer.

Ado-trastuzumab emtansine (Kadcyla) is a treatment for advanced HER2-positive breast cancer got approved by FDA.

Lapatinib (Tykerb) got approved for treatment of HER2-positive metastatic BC. However, in another study it doesn't show significant effect. 
Neratinib Maleate (Nerlynx) can be used in patients with earlystage HER2-positive BC and also can be used in combination with capecitabine (Xeloda) in some patients in advanced stage.

\section{Triple-negative breast cancer}

Due to the lack of HR and HER2 over expression triple negative $\mathrm{BC}$ is hardest of all PARP inhibitors, which include olaparib (Lynparza) and talazoparib (Talzenna), effectively target TNBC caused by different DNA mutation and damage. They also got approved for the treatment of metastatic ER-positive, HER2-negative BC in patients with gene mutation.

Immunotherapy drugs have shown effectiveness in small number of patients, Mainly those that are triple negative. Studies suggest that the immune therapy is more effective when the patients have their tumor expresses the protein PD-L1 or if it has a large number of mutations.

Studies are conducted to check the effectiveness of combination therapy. The immunotherapy drug atezolizumab (Tecentriq) got approved for using in combination with chemotherapy in patients with metastatic TNBC that expresses the PD-L1 protein [320].

\section{Conclusion}

Breast cancer classified in to 3 major tumor subtypes which is categorized accordingly to estrogen or progesterone receptor expression and HER2 expression. These 3 subtypes have their own distinct risk profiles and treatment strategies. Individualized therapy for each patient depends on tumor subtype, anatomic cancer stage and patients preferences are required or better therapeutic response [3].

\section{Bibliography}

1. Siegel Rebecca L., et al. "Cancer statistics, 2020". CA: A Cancer Journal for Clinicians 70.1 (2020): 7-30.

2. National Institutes of Health; National Cancer Institute. "Surveillance, Epidemiology, and End Results Program". Cancer stat facts: female breast cancer.

3. Waks Adrienne G and Eric P Winter. "Breast cancer treatment: a review". The Journal of the American Medical Association 321.3(2019): 288-300.

4. Joshi H and Press MF. "Molecular oncology of breast cancer". In: Bland KI, Copeland EM, Klimberg VS, Gradishar WJ, editions. The Breast. Philadelphia, PA: Elsevier (2018): 22.

5. Denkert C., et al. "Molecular alterations in triple-negative breast cancer-the road to new treatment strategies". Lancet 389.10087 (2017): 2430-2442.

6. Chavez-MacGregor M., et al. "Incorporating tumor characteristics to the American Joint Committee on Cancer Breast Cancer Staging System". Oncologist 22.11 (2017): 1292-1300.
7. Bardia A., et al. "Efficacy and Safety of anti-trop-2 antibody drug conjugate sacituzumab govitecan (IMMU-132) in heavily pretreated patients with metastatic triple-negative breast cancer". Journal of Clinical Oncology 35.19 (2017): 2141-2148.

8. Francis PA., et al. "SOFT and TEXT Investigators and the International Breast Cancer Study Group. Tailoring adjuvant endocrine therapy for premenopausal breast cancer". The New England Journal of Medicine 379.2 (2018): 122-137.

9. Pan H., et al. "EBCTCG. 20-Year risks of breast-cancer recurrence after stopping endocrine therapy at 5 years". The New England Journal of Medicine 377.19 (2017): 1836-1846.

10. Goss PE., et al. "Extending aromatase-inhibitor adjuvant therapy to 10 years". The New England Journal of Medicine 375.3 (2016): 209-219.

11. Gluz O., et al. "West German Study Group Phase III PlanB Trial: first prospective outcome data for the 21-gene recurrence score assay and concordance of prognostic markers by central and local pathology assessment". Journal of Clinical Oncology 34.20 (2016): 2341-2349.

12. Sparano JA., et al. "Adjuvant chemotherapy guided by a 21-gene expression assay in breast cancer". The New England Journal of Medicine 379.2 (2018): 111-121.

13. Jasem J., et al. "The 21-gene recurrence score assay for nodepositive, early-stage breast cancer and impact of RxPONDER Trial on chemotherapy decision-making: have clinicians already decided?" Journal of the National Comprehensive Cancer Network 15.4 (2017): 494-503.

14. Cardoso F., et al. "MINDACT Investigators. 70-Gene signature as an aid to treatment decisions in early-stage breast cancer". The New England Journal of Medicine 375.8 (2016): 717-729.

15. Harris LN., et al. "American Society of Clinical Oncology. Use of biomarkers to guide decisions on adjuvant systemic therapy for women with early-stage invasive breast cancer: American Society of Clinical Oncology Clinical Practice Guideline". Journal of Clinical Oncology 34.10 (2016): 1134-1150.

16. Krop I., et al. "Use of biomarkers to guide decisions on adjuvant systemic therapy for women with early-stage invasive breast cancer: American Society of Clinical Oncology clinical practice guideline focused update". Journal of Clinical Oncology 35.24 (2017): 2838-2847.

17. Duffy MJ., et al. "Clinical use of biomarkers in breast cancer: updated guidelines from the European Group on Tumor Markers (EGTM)". European Journal of Cancer 75 (2017): 284-298.

18. Masuda N., et al. "Adjuvant capecitabine for breast cancer after preoperative chemotherapy". The New England Journal of Medicine 376.22 (2017): 2147-2159. 
19. Cameron D., et al. "Herceptin Adjuvant (HERA) Trial Study Team. 11 Years' follow-up of trastuzumab after adjuvant chemotherapy in HER2-positive early breast cancer: final analysis of the HERceptin Adjuvant (HERA) trial". Lancet 38910075 (2017): 1195-1205.

20. Earl HM., et al. "PERSEPHONE: 6 versus 12 months (m) of adjuvant trastuzumab in patients (pts) with HER2 positive (+) early breast cancer (EBC): randomised phase 3 non-inferiority trial with definitive 4-year (yr) disease-free survival (DFS) results". Journal of Clinical Oncology 36.15 (2018).

\section{Assets from publication with us}

- Prompt Acknowledgement after receiving the article

- Thorough Double blinded peer review

- Rapid Publication

- Issue of Publication Certificate

- High visibility of your Published work

Website: https://www.actascientific.com/

Submit Article: https://www.actascientific.com/submission.php Email us: editor@actascientific.com

Contact us: +919182824667 